Supporting Information for:

\title{
Electrochemical Nanoneedle Biosensor based on Multiwall Carbon Nanotube
}

Hankil Boo, ${ }^{\dagger, \ddagger}$ Ran-A Jeong, ${ }^{\dagger, \ddagger}$ Sejin Park, ${ }^{\dagger, \ddagger}$ Keun Soo Kim, ${ }^{\S}$ Kay Hyeok An, ${ }^{\S}$ Young Hee Lee, ${ }^{\S}$ Ji Hyung Han, ${ }^{\dagger}$ Taek Dong Chung ${ }^{\dagger} *$ and Hee Chan $\mathrm{Kim}^{\ddagger} *$

${ }^{\dagger}$ Center for NanoBio Applied Technology, Sungshin Women’s University, 249-1

Dongsun-dong, Seongbuk-gu, Seoul 136-742, Republic of Korea

${ }^{\ddagger}$ Department of Biomedical Engineering, College of Medicine and Institute of Medical and Biological Engineering, Medical Research Center, Seoul National University, 28 Yongon-dong, Chongno-gu, Seoul 110-744, Republic of Korea

${ }^{\S}$ Department of Physics, Center for Nanotubes and Nanostructured Composites, Sungkyunkwan University, Suwon 440-746, Republic of Korea

${ }^{\dagger}$ Tel: +82-2-920-7361, Fax: +82-2-922-0760, E-mail: chembud@sungshin.ac.kr

${ }^{\ddagger}$ Tel: +82-2-760-2931, Fax: +82-2-, E-mail: hckim@snu.ac.kr 
Manufacture of CNT nanoneedles: The nanoneedle-supporitng tip material is a tungsten wire (diam. $0.25 \mathrm{~mm}$ length $25 \mathrm{~mm}$ ). Etching parameters were optimized to archieve the desired tip aspect ratio. The etching media were $1 \mathrm{M}$ and $2 \mathrm{M}$ sodium hydroxide $(\mathrm{NaOH})$ aqueous solutions. The bias voltages of $5 \mathrm{~V}$ and $10 \mathrm{~V}$ were applied. The emersion depth was varied $3 \mathrm{~mm}$ and $10 \mathrm{~mm}$.

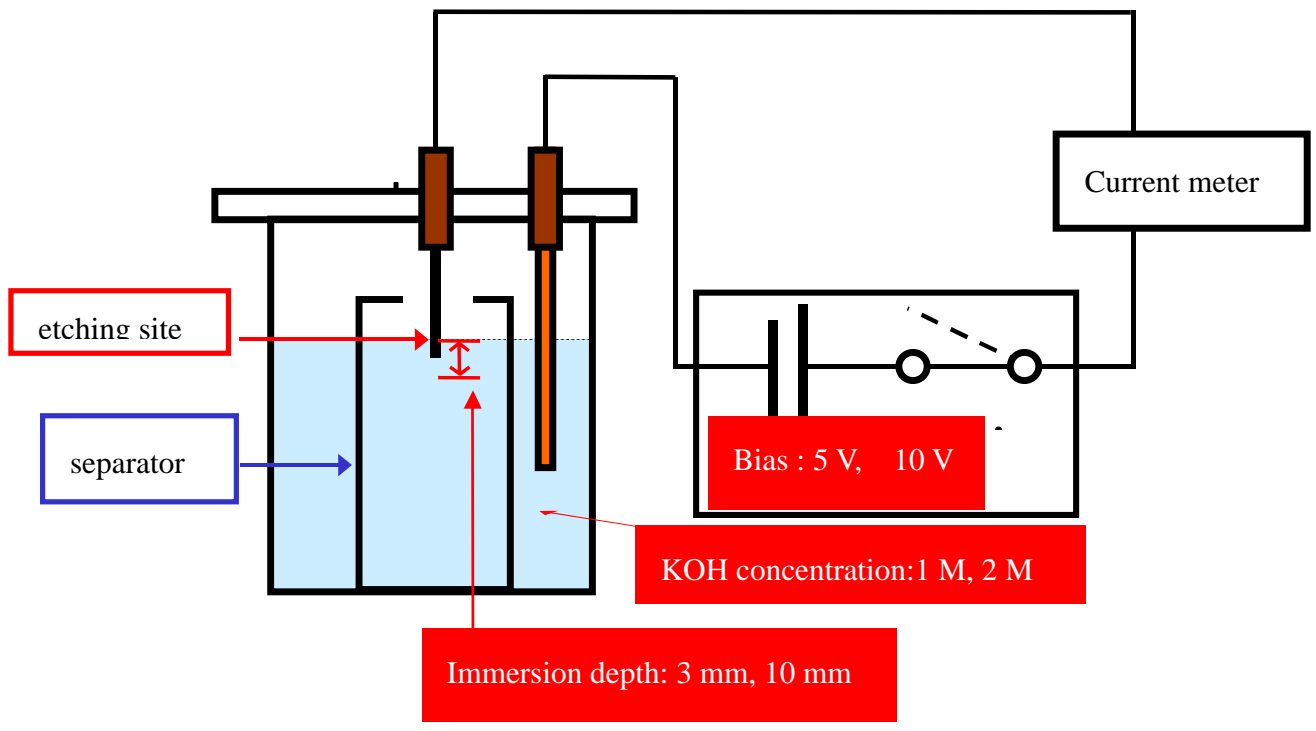

Figure 1. Schematic diagram of the tungsten etching setup.
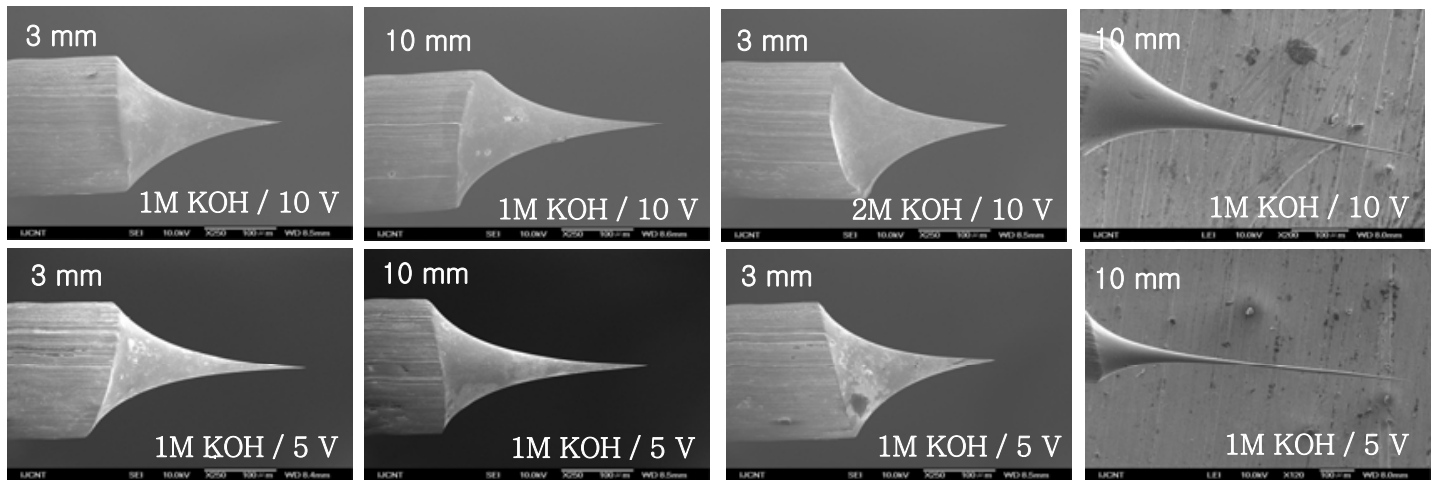

Figure 2. The effect of etching conditions on the aspect ratio of tungsten tip. 
The aspect ratio is predominantly influenced by immersion depth. And the low bias voltage contributes a little to further sharpening of the tip. The result is shown in Figure 2. The etching process was followed by dipping the tip in $\mathrm{HF}(49 \%)$ solution for 5 second and then rinsing it with deionized water for 1 hour to remove the residual $\mathrm{NaOH}$ on tungsten tip. In the vaccuum chamber of the field emission-scanning electron microscope (FE-SEM), the end of the tungsten tip is touched to an individual MWCNT out of the bundle of carbon nanotubes. After locating the MWCNT it was pulled out of the bundle, a sufficient voltage is applied, then a weak site of the carbon nanotube is cutted and the length is $2 \sim 3 \mu \mathrm{m}$ as displayed in Figure 1 of Text. Figure 3 shows that the current drop is observed when the carbon nanotube is cut.

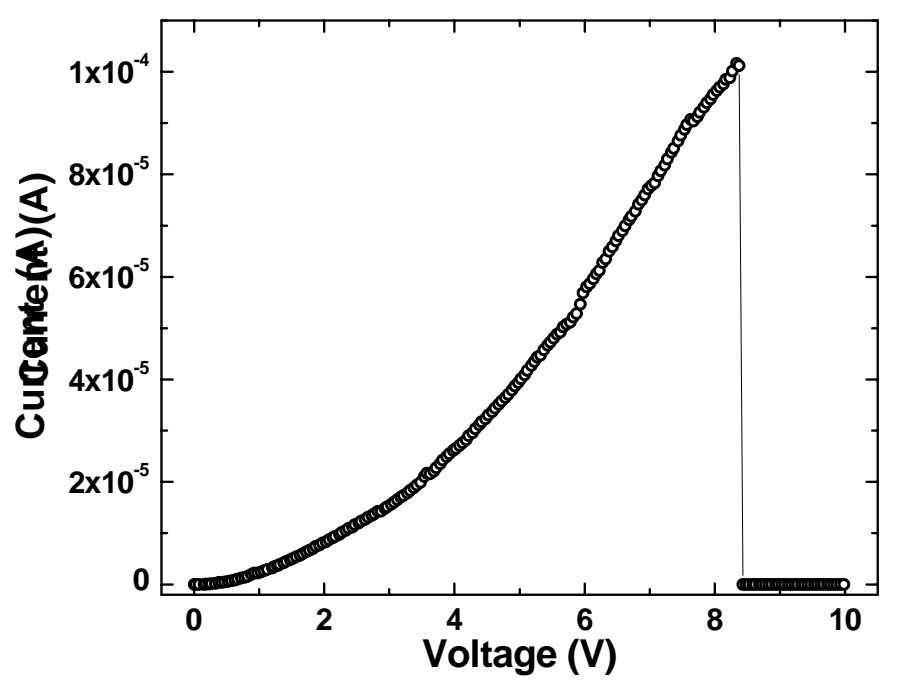

Figure 3. Current change vs. applied voltage during the carbon nanotube cutting. 
Confirmation of sensitivity of a carbon nanotube as a biosensor: The possibility of exposure of tungsten still exists despite insulating procedure. Thus it is necessary to confirm whether the electrochemical signals are due to the tungsten or the carbon nanotube. Figure 4 is a differential pulse voltammogram of dopamine in HEPES buffer solution from the tungsten tip only that was insulated except the end of the tip. No dopamine oxidation and only a small background current were observed, which contrasted with the results obtained using a nanoneedle (Figure 3 in Text). Therefore we can see that the electrochemical signals of dopamine oxidation come from the carbon nanotube.

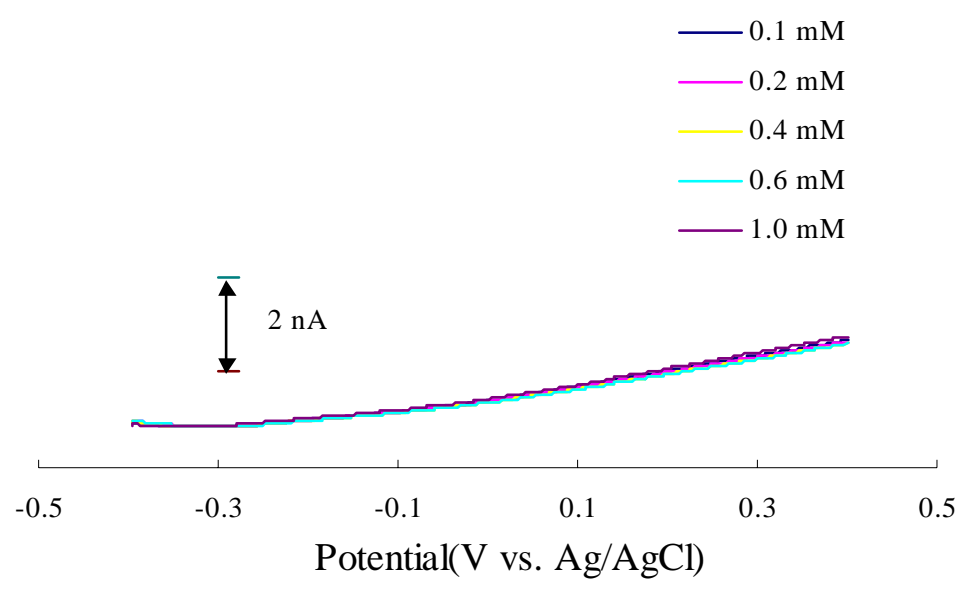

Figure 4. Differential pulse voltammograms of dopamine oxidation at only tungsten tip in a HEPES solution. 